\title{
Manet Routing Protocols on Network LAYER IN REALTIME SCENARIO
}

\author{
Mamata Rath*, Binod Kumar Pattanayak** and Bibudhendu Pati*** \\ *C.V.Raman Computer Academy,Bhubaneswar,India \\ **Department of Computer Science and Engineering, Siksha 'O' Anusandhan University, \\ Bhubaneswar,India \\ ***Department of Computer Science and Engineering, C. V. Raman College of \\ Engineering, Bhubaneswar,India
}

\begin{abstract}
A Mobile Ad hoc Network comprises of a group of mobile nodes that are connected in wireless medium and they dynamically form a self-organizing mobile network for temporary period of time. Due to ease of deployment, MANET is very effective in situations where it is difficult to create infrastructure based network. Nodes in a MANET are self configured and self governed without being controlled by any central administrator. Mobile Adhoc Network (MANET) has always been a rising technology which supports real time multimedia transmission service, a common trend in current communication technology that uses maximum real time applications with dynamic mobility of the mobile devices. Offering Quality of Service is also an important parameter during communication in real time scenario.Many routing protocols for Mobile Adhoc Network Supporting Real Time Applications have been developed, basic intention being maximum utilization of resource in resource constraint environment, minimum power consumption using limited residual battery power of the highly movable mobile nodes. This paper presents an intensive study and analysis of MANET based effective routing protocols and secondly, it provides report on comparative analysis of efficient real time supported protocols based on power consumption, delay analysis and packet delivery ratio.
\end{abstract}

\section{KEYWORDS}

MANET,AODV,PDR,Throughput,Delay, Real Time Appliction

\section{INTRODUCTION}

Real time data transmission in Mobile Adhoc Network is a challenging issue due to the selfdirected nature of mobile devices which are connected with each other by wireless links. Nodes frequently changes their position and location by forming a network without any base or any infrastructure. Careful considerations have to be made during designing the protocols for real time data transmission which are highly sensitive. Common challenges faced by real time data during transmission are energy utilization, scalability of the network, optimized use of bandwidth, resource reservation, reducing chance of link failure, security, take care of Qos, dynamically changing topology etc.

\section{LITERATURE REVIEW}

Many researchers have done analysis e regarding successful transmission of real time data over MANETs, some of which are discussed in this section. Scheduling algorithms basically classifies 
and categorizes high priority-based real time applications to enhance the efficient delivery of realtime packets in the channel. In paper[11] five types of such scheduling algorithms are examined under real time traffic condition. They are First-In-First-Out (FIFO) Scheduling Algorithm, Priority Queuing Algorithm, Weighted Fair Queuing (WFQ) Algorithm, Class Based Weighted Fair Queuing (CBWFQ) Algorithm and Low Latency Queuing (LLQ) Algorithm. After simulation was done in OPNET, the results were compared and evaluated for the following parameters for the above five algorithms.a.Voice traffic receivedb. video traffic receivedc. voice packet end-to-end delay d. voice packet delay variation.In every case the performance of LLQ algorithm was found to be better with lower delay and higher throughput.

In a dense MANET scenario, where the source is situated far away from the destination, proper data delivery for real-time video streaming is a difficult task. In video traffic flows which is too heavy and need to be continuously flowed without interruption, so sending packets continuously without pause causes congestion of its own flow.In [3] the authors propose a method of periodically delaying the realtimedata transmission specifically video stream at source itself just to avoid more congestion at the next-hop relaying node level.

\section{Real Time Traffic and Routing Protocols}

Detail study and analysis of Real Time Protocols have been done in this section. Table 1. Provides description of studied protocols and analysis based on Real Time Support.

Table.1 Details of studied and analyzed Protocols

\begin{tabular}{|c|c|c|c|c|c|c|}
\hline $\begin{array}{l}\text { Sl.N } \\
\text { o }\end{array}$ & Literature & $\begin{array}{l}\text { Yea } \\
\mathrm{r}\end{array}$ & Protocol/Proposal & Feature & Advantage & $\begin{array}{l}\text { Simulation } \\
\text { Tool }\end{array}$ \\
\hline 1 & $\begin{array}{l}\text { D.Tardioli } \\
\text {, et al [1] }\end{array}$ & $\begin{array}{l}201 \\
5\end{array}$ & $\begin{array}{l}\text { RT-WMP } \\
\text { Real Time } \\
\text { Wireless Manet } \\
\text { Protocol }\end{array}$ & $\begin{array}{l}\text { Proposes a cross- } \\
\text { layer strategy } \\
\text { with a novel } \\
\text { MAC } \\
\text { mechanism }\end{array}$ & $\begin{array}{l}\text { Priority ad mobility } \\
\text { support for real-time } \\
\text { communications }\end{array}$ & $\begin{array}{l}\text { Real-world } \\
\text { application } \\
\text { Linux Kernel }\end{array}$ \\
\hline 2 & $\begin{array}{l}\text { H.Xie, et } \\
\text { al [2] }\end{array}$ & $\begin{array}{l}201 \\
5\end{array}$ & $\begin{array}{l}\text { An error-recovery } \\
\text { mechanism is } \\
\text { proposed for real- } \\
\text { time video } \\
\text { streaming called } \\
\text { MERVS }\end{array}$ & $\begin{array}{l}\text { Transmission of } \\
\text { video through } \\
\text { multiple } \\
\text { channels and } \\
\text { priority queue is } \\
\text { used }\end{array}$ & $\begin{array}{l}\text { High quality video } \\
\text { streaming with FEC } \\
\text { (Forward Error } \\
\text { Correction) }\end{array}$ & NS2 \\
\hline 3 & $\begin{array}{l}\text { P.Sondi, } \\
\text { et al [3] }\end{array}$ & $\begin{array}{l}201 \\
4\end{array}$ & $\begin{array}{l}\text { A new MANET } \\
\text { based strategy to } \\
\text { handle real time } \\
\text { application is } \\
\text { presented }\end{array}$ & $\begin{array}{l}\text { At source } \\
\text { periodical delay } \\
\text { is maintained to } \\
\text { avoid congestion } \\
\text { and hence avoids } \\
\text { interruption }\end{array}$ & $\begin{array}{l}30 \% \text { improvement } \\
\text { in delivery of video } \\
\text { packets between } \\
\text { source and } \\
\text { destination }\end{array}$ & OPNET \\
\hline 4 & $\begin{array}{l}\text { G.Yasin, } \\
\text { et al }[4]\end{array}$ & $\begin{array}{l}201 \\
3\end{array}$ & $\begin{array}{l}\text { Performance } \\
\text { comparison of } \\
\text { protocols }\end{array}$ & $\begin{array}{l}\text { Study of } \\
\text { different routing } \\
\text { protocols, } \\
\text { specifically } \\
\text { OLSR,AODV,D } \\
\text { SR, } \\
\text { TORA }\end{array}$ & $\begin{array}{l}\text { Gives idea about } \\
\text { the routing nature } \\
\text { for real time traffic }\end{array}$ & OPNET \\
\hline 5 & $\begin{array}{l}\text { I.Ahmad, } \\
\text { et al [5] }\end{array}$ & $\begin{array}{l}201 \\
3\end{array}$ & $\begin{array}{l}\text { TSBR-Time Slot } \\
\text { Bandwidth } \\
\text { Reservation }\end{array}$ & $\begin{array}{l}\text { Presents a } \\
\text { technique that } \\
\text { provides better } \\
\text { QoS using time- } \\
\text { slot bandwidth }\end{array}$ & $\begin{array}{l}\text { Reduces queuing } \\
\text { delay }\end{array}$ & NS2 \\
\hline
\end{tabular}


International Journal on Cybernetics \& Informatics (IJCI) Vol. 5, No. 1, February 2016

\begin{tabular}{|c|c|c|c|c|c|c|}
\hline & & & & $\begin{array}{l}\text { reservation } \\
\text { method }\end{array}$ & & \\
\hline 6 & $\begin{array}{l}\text { P.Vijaya } \\
\text { Kumar, et } \\
\text { al [6] }\end{array}$ & $\begin{array}{l}201 \\
3\end{array}$ & $\begin{array}{l}\text { A Multicast tree } \\
\text { structure protocol }\end{array}$ & $\begin{array}{l}\text { Challenge of } \\
\text { transmission of } \\
\text { multimedia } \\
\text { stream is } \\
\text { investigated }\end{array}$ & $\begin{array}{l}\text { A new technique for } \\
\text { multimedia stream } \\
\text { is presented here }\end{array}$ & NS2 \\
\hline 7 & $\begin{array}{l}\text { Z.Yuan, } \\
\text { et al [7] }\end{array}$ & $\begin{array}{l}201 \\
3\end{array}$ & $\begin{array}{l}\text { iPAS-intelligent } \\
\text { Prioritized } \\
\text { Adaptive Scheme }\end{array}$ & $\begin{array}{l}\text { Mainly discusses } \\
\text { suitable routing } \\
\text { method for } \\
\text { multimedia data }\end{array}$ & $\begin{array}{l}\text { Proposes an } \\
\text { efficient scheme } \\
\text { called } \\
\text { iPAS(Intelligent } \\
\text { Prioritised Adaptive } \\
\text { Scheme) }\end{array}$ & $\begin{array}{l}\text { Real-life test } \\
\text { bed } \\
\text { set up with } \\
\text { multimedia } \\
\text { server }\end{array}$ \\
\hline 8 & $\begin{array}{l}\text { R.Viegas, } \\
\text { et al }[8]\end{array}$ & $\begin{array}{l}201 \\
3\end{array}$ & $\begin{array}{l}\text { A new Real time } \\
\text { communication } \\
\text { scheme }\end{array}$ & $\begin{array}{l}\text { Proposes a new } \\
\text { idea to prioritize } \\
\text { real time flow in } \\
\text { a network }\end{array}$ & $\begin{array}{l}\text { Proves better } \\
\text { throughput for real } \\
\text { time data flow }\end{array}$ & NS2 \\
\hline 9 & $\begin{array}{l}\text { D.Sicigna } \\
\text { no, et al } \\
{[9]}\end{array}$ & $\begin{array}{l}201 \\
3\end{array}$ & $\begin{array}{l}\text { Real time multi- } \\
\text { hop protocol with } \\
\text { Qos extension }\end{array}$ & $\begin{array}{l}\text { Proposes the use } \\
\text { of multi-hop } \\
\text { adhoc network } \\
\text { to provide } \\
\text { multimedia } \\
\text { communication } \\
\text { between mobile } \\
\text { nodes }\end{array}$ & $\begin{array}{l}\text { Use of low cost s/w } \\
\text { and h/w platform to } \\
\text { run the protocol, } \\
\text { easy to set up } \\
\text { solution. }\end{array}$ & $\begin{array}{l}\text { Real } \\
\text { application in } \\
\text { Somport } \\
\text { tunnel, } \\
\text { France }\end{array}$ \\
\hline 10 & $\begin{array}{l}\text { J.Boksine } \\
\text { r, et al } \\
{[10]}\end{array}$ & $\begin{array}{l}201 \\
3\end{array}$ & $\begin{array}{l}\text { MRFM-MANET } \\
\text { Real time } \\
\text { Frequency } \\
\text { Management } \\
\text { approach is } \\
\text { proposed }\end{array}$ & $\begin{array}{l}\text { Frequency re-use } \\
\text { automation, and } \\
\text { spectrum sharing } \\
\text { with centralized } \\
\text { control }\end{array}$ & $\begin{array}{l}\text { Dynamic Spectrum } \\
\text { Access capability } \\
\text { among MANET } \\
\text { radio layer }\end{array}$ & $\begin{array}{l}\text { Real } \\
\text { implementati } \\
\text { on in android } \\
\text { device }\end{array}$ \\
\hline 11 & $\begin{array}{l}\text { P.Rukman } \\
\text { i, et al } \\
{[11]}\end{array}$ & $\begin{array}{l}201 \\
3\end{array}$ & $\begin{array}{l}\text { Analysis of five } \\
\text { scheduling } \\
\text { algorithm for real } \\
\text { time transmission }\end{array}$ & $\begin{array}{l}\text { Voice and video } \\
\text { traffic simulated } \\
\text { using WFQ \& } \\
\text { LLQ algorithm. }\end{array}$ & $\begin{array}{l}\text { LLQ(Low Latency } \\
\text { Queuing ) algorithm } \\
\text { improves the overall } \\
\text { performance of real } \\
\text { time applications }\end{array}$ & OPNET \\
\hline 12 & $\begin{array}{l}\text { H.Mewar } \\
\text { a, et al } \\
{[12]}\end{array}$ & $\begin{array}{l}201 \\
3\end{array}$ & $\begin{array}{l}\text { Evaluation of } \\
\text { protocols based } \\
\text { on Pattern of } \\
\text { traffic and Node } \\
\text { density }\end{array}$ & $\begin{array}{l}\text { AODV,DSR,OL } \\
\text { SR,TORA are } \\
\text { evaluated for } \\
\text { delay, n/w load, } \\
\text { throughput }\end{array}$ & $\begin{array}{l}\text { Gives a better } \\
\text { knowledge of } \\
\text { protocol selection } \\
\text { for Qos-aware } \\
\text { traffic. }\end{array}$ & $\begin{array}{l}\text { OPNET } \\
\text { Ver } 14.5\end{array}$ \\
\hline 13 & $\begin{array}{l}\text { G.Aujla, } \\
\text { et al [13] }\end{array}$ & $\begin{array}{l}201 \\
3\end{array}$ & $\begin{array}{l}\text { Evaluation of } \\
\text { Behavior of } \\
\text { GRP,TORA,OLS } \\
\text { R protocols for } \\
\text { video } \\
\text { conferencing } \\
\text { application }\end{array}$ & $\begin{array}{l}\text { Simulation } \\
\text { shows that } \\
\text { TORA has } \\
\text { highest } \\
\text { throughput in } \\
\text { most scenarios. }\end{array}$ & $\begin{array}{l}\text { Better analysis of } \\
\text { protocols to select a } \\
\text { suitable one for real } \\
\text { time transmission. }\end{array}$ & $\begin{array}{l}\text { OPNET } \\
\text { Modeler } \\
14.5\end{array}$ \\
\hline 14 & $\begin{array}{l}\text { C.Lal, et } \\
\text { al [14] }\end{array}$ & $\begin{array}{l}201 \\
3\end{array}$ & $\begin{array}{l}\text { QARP-Qos } \\
\text { Aware Routing } \\
\text { Protocol using } \\
\text { cross-layer design } \\
\text { proposed }\end{array}$ & $\begin{array}{l}\text { Session } \\
\text { Admission } \\
\text { Control method } \\
\text { is used in the } \\
\text { protocol }\end{array}$ & $\begin{array}{l}\text { Normal periodic } \\
\text { message format can } \\
\text { be extended to } \\
\text { minimize the effect } \\
\text { of mobility }\end{array}$ & NA \\
\hline
\end{tabular}


In paper [9] a survey of multi-path video transmission in MANETs has been done. Parameters which were considered during the analysis were Coding methods, Optimal number of streams, video data rate, multi-path routing etc. A coding intrusive algorithm was presented for choosing optimal parameters for successful "network friendly" video transmission over MANET.

\section{Comparative Analysis Of Realtime Protocols}

Three important real time supported protocols were selected for their performance comparison and analysis to highlight on the functionality used by them in order to improve the network performance in real time scenario. They are RT-WMP[1], MCT[6] and MRFM [10] approach with Real Time Support.

Table 2. Describes studied performance of the three selected protocols.

Table 2. Comparison of Network Performance Parameters in three leading protocols

\begin{tabular}{|c|c|c|c|c|c|}
\hline Protocol & $\begin{array}{c}\text { Power } \\
\text { Consumed }\end{array}$ & $\begin{array}{c}\text { End to end } \\
\text { delay }\end{array}$ & $\begin{array}{c}\text { Network } \\
\text { Life time }\end{array}$ & PDR & $\begin{array}{c}\text { QoS } \\
\text { Support }\end{array}$ \\
\hline RT WMP & Low & Low & More & High & Medium \\
\hline MCT & Medium & Medium & Medium & $\begin{array}{c}\text { Medi } \\
\text { um }\end{array}$ & Low \\
\hline MRFM & High & High & Low & low & High \\
\hline
\end{tabular}

\section{Simulation AND RESUlt}

We have done simulation of above considered protocols for their performance evaluation based on Packet Delivery Ratio, Power Consumption, Delay and Network Lifetim .Ns 2.35 simulation tool was used for simulation purpose. 120 number of nodes were scattered randomly in an area of $2000 \times 2000 \mathrm{~m}$ during beginning of the simulation. In mobility scenario, mobiles nodes were moving in six different speeds within a range of 0 to $10 \mathrm{~m} / \mathrm{s}$. Table 3 shows the simulation parameters used in the simulation.

Table 3. Simulation Parameters

\begin{tabular}{|l|l|}
\hline Simulation parameters & Data/Value \\
\hline Channel Type & Wireless Channel \\
\hline MAC Protocol & 802.11 \\
\hline Traffic Type & CBR \\
\hline Packet Size & 512 Bytes \\
\hline No. of Mobile Nodes & 120 \\
\hline Simulation Time & 600 seconds $(10$ mins $)$ \\
\hline Simulation area & 2000 x $2000 \mathrm{~m}$ \\
\hline Protocols Name & RTWMP,MCT,MRFM \\
\hline Model for Node Movement & Random way point \\
\hline Data rate & 11 mbps \\
\hline Application Type & Video Transmission (Real Time) \\
\hline Bandwidth & $2 \mathrm{Mb} / \mathrm{s}$ for both \\
\hline Simulator & Ns2.35 \\
\hline &
\end{tabular}


Comparative analysis of the three discussed protocols has been done in the following section based on network parameters such as power consumption, delay rate, packet delivery ratio and network lifetime.

Fig.1 shows the comparison between three Protocols for packet delivery ratio nd it was observed that RTWMP performs better than MCT and MRFM.

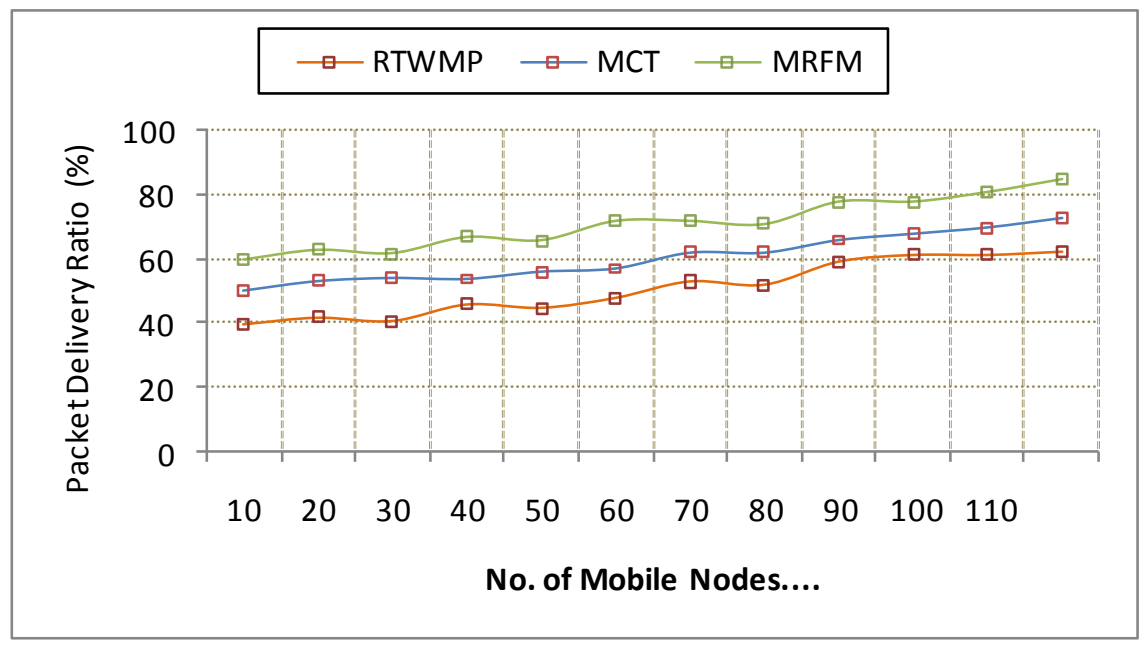

Fig.1 PDR Analysis in Video Conference Application

\section{CONCLUSiON}

The above discussed theme first presents detailed study on challenging protocols, highlights on the mechanisms usded by important real time based routing protocols in MANET and some analysis which has been done to evaluate their performance. Secondly, three leading protocols are selecetd based on specturm distribution in the wireless channel and on the basis of their technical mechanism and approches for comparative analysis, the result of simulation are presented which we believe will be useful for further analysis and development of a robust and versatile realtime based protocol for the magnificent area of MANET.

\section{REFERENCE}

[1] DaniloTardioli ,DomenicoSicignano , José Luis Villarroel,(2015)” A wireless multi-hop protocol for real-time applications",Computer Communications, Vol 55, Pages-4-21.

[2] Xie, H., Boukerche, A., Loureiro, A.,(2015) "MERVS: A Novel Multi-channel Error Recovery Video Streaming Scheme for Vehicle Ad-hoc Networks," Vehicular Technology, IEEE Transactions on ,Vol 99.

[3] Sondi, P., Gantsou, D.,(2014) "Improving real-time video streaming delivery over dense multi-hop wireless ad hoc networks," Wireless Days (WD), 2014 IFIP, Pages 1-4.

[4] GhulamYasin, Syed Fakhar Abbas, S. R. Chaudhry, (2013)“ MANET Routing Protocols for RealTime Multimedia Applications," WSEAS Transactions on Communications, Vol 12, Issue 8, Pages 386-395.

[5] Ahmad, Iftikhar, SamreenAyaz, Syed Yasser Arafat, Faisal Riaz, and HumairaJabeen,(2013) "QoS routing for real time traffic in mobile ad hoc network", Proceedings of the 7th International Conference on Ubiquitous Information Management and Communication.

[6] P.D.R. Vijaya Kumar and T. Ravichandran,(2013) “ A Real Time Multimedia Streaming in Mobile Ad Hoc Networks using Multicast Tree Structure" Research Journal of Information Technology, Vol 5,Issue 1,Pages 24-34. 
[7] Zhenhui Yuan, and Gabriel-MiroMuntean,(2013)" A Prioritized Adaptive Scheme for Multimedia Services over IEEE 802.11 WLANs," IEEE Transactions on Network and Service Management, Vol. 10, No. 4.

[8] RaimundoViegas, Jr., LuizAffonsoGuedes , (2013) Francisco Vasques , Paulo Portugal , Ricardo Moraes "A new MAC scheme specifically suited for real-time industrial communication based on IEEE 802.11e" ELSEVIER, Computers and Electrical Engineering, Pages-1684-1704.

[9] D. Sicignano, D. Tardioli, S. Cabrero, J.L. Villarroel,(2013) " Real-time wireless multi hop protocol in underground voice communication," Ad Hoc Networks Vol.11,Issue 4, Pages- 1484-1496.

[10] Boksiner, J., Posherstnik, Y., May, B., Saltzman, M., Kamal, S.,(2013) "Centrally Controlled Dynamic Spectrum Access forMANETs,", Military Communications Conference, MILCOM 2013 2013 IEEE ,Pages 641-646, 18-20.

[11] P.Rukmani,R.Ganesan, (2013) "Scheduling algorithm for real time applications in mobile ad hoc network with opnet modeler," International Conference On Design And Manufacturingg, IConDM 2013,Procedia Engineering, Vol 64, Pages. 94 - 103.

[12] Mewara, H.S., Porwal, S.,(213) "Node density and traffic based modelling and analysis of routing protocols for MANETs," Advances in Technology and Engineering (ICATE), 2013 International Conference on ,Pages 1-6.

[13] G. S. Aujla, S. S. Kang,(2013) "Simulation based comparative analysis of TORA, OLSR and GRP routing protocols for email and video conferencing applications over MANETs", International Journal of Computer Networking Wireless and Mobile Communications Vol. 3, Pages- 353-362.

[14] Lal, C., Laxmi, V., Gaur, M.S.,(2013) "QoS-aware routing for transmission of H.264/SVC encoded video traffic over MANETs," Communications (APCC), 2013 19th Asia-Pacific Conference on , Pages 104-109. 